\title{
Realization and high power test of damped $C$-band accelerating structures
}

\author{
D. Alesini $\odot$, M. Bellaveglia, F. Cardelli, R. Di Raddo, \\ A. Gallo, V. Lollo, L. Piersanti, and A. Variola \\ Laboratori Nazionali di Frascati, INFN, Via Enrico Fermi, 54 (già 40), 00044 Frascati, Rome, Italy \\ L. Palumbo \\ University of Rome La Sapienza and INFN - Romal, 00185 Rome, Italy \\ F. Poletto and P. Favaron \\ Laboratori Nazionali di Legnaro, INFN, Viale dell'Università 2, 35020 Legnaro, Padua, Italy
}

(Received 19 May 2019; revised manuscript received 10 January 2020; accepted 18 February 2020; published 6 April 2020)

\begin{abstract}
The linac of the European project Extreme Light Infrastructure-Nuclear Physics (ELI-NP) foresees the use of 12 traveling wave $C$-band accelerating structures. The cavities are $1.8 \mathrm{~m}$ long, quasiconstant gradient, and have a field phase advance per cell of $2 \pi / 3$. They operate at $100 \mathrm{~Hz}$ repetition rate, and, because of the multibunch operation, they have been designed with a dipole higher-order mode (HOM) damping system to avoid beam breakup. The structures have symmetric input and output couplers and integrate, in each cell, a damping system based on silicon carbide (SiC) rf absorbers coupled to each cell through waveguides. An optimization of the electromagnetic and mechanical design has been done to simplify the fabrication and to reduce the costs. The cavities have been fabricated, and the first full-scale prototype has been also successfully tested at the nominal gradient of $33 \mathrm{MV} / \mathrm{m}$, repetition rate of $100 \mathrm{~Hz}$, and pulse length of $820 \mathrm{~ns}$. It represents, to our knowledge, the first full-scale linac structure with HOM damping waveguides and $\mathrm{SiC}$ absorbers tested at this high gradient. In the paper, we illustrate the realization process of such a complicated device together with the low and high power test results.
\end{abstract}

DOI: 10.1103/PhysRevAccelBeams.23.042001

\section{INTRODUCTION}

The gamma-ray beam system of the European project Extreme Light Infrastructure-Nuclear Physics (ELI-NP) [1] foresees the use of a multibunch train of electrons colliding with a high-intensity recirculated laser pulse [2], thus generating an intense source of gamma-ray photons by Compton backscattering. The 32 electron bunches, each carrying $250 \mathrm{pC}$ of charge and separated by $16 \mathrm{~ns}$, will be accelerated in an rf pulse at a repetition rate of $100 \mathrm{~Hz}$. The linac consists of an $S$-band injector and 12 traveling wave (TW) $C$-band disk loaded accelerating structures, $1.8 \mathrm{~m}$ long, quasiconstant gradient with $2 \pi / 3$ field phase advance per cell operating at $33 \mathrm{MV} / \mathrm{m}$ average accelerating field. Since the linac is operated in multibunch mode, the $C$-band structures have been designed with a heavy damping of the higher-order dipole modes (HOM) based on waveguide coupling to $\mathrm{SiC}$ absorbers.

$C$-band accelerating structures have been already designed and fabricated for different projects

Published by the American Physical Society under the terms of the Creative Commons Attribution 4.0 International license. Further distribution of this work must maintain attribution to the author(s) and the published article's title, journal citation, and DOI.
[3-9]. Those implemented for SPARC_LAB, SwissFel, and SXFEL in Shanghai Institute of Applied Physics [7-9] are iris-loaded structures, without damping, because they operate in single-bunch mode. The Spring 8 ones [3-6] are, indeed, choke mode-based damped structures, but those we developed for the ELI-NP structures [10] exhibit a higher shunt impedance and can operate at a higher repetition rate with an higher average dissipated power. The design criteria of these cavities have been already illustrated in Ref. [10]. In the present paper, we present how such complicated structures were realized with showing the detail of the fabrication mostly in the assembly stage, the low and high power test result including tuning, and vacuum measurement results. To our knowledge, these cavities have been the first full-scale waveguide damped structures to be tested at such a high gradient [11]. The main structures parameters are given in Table I. The sketch of the cavities is reported in Fig. 1. The cell damping system is similar to the design adopted for the Compact Linear Collider (CLIC) $X$-band structures [12-14], but the mechanical design has been strongly simplified to reduce the cost and to simplify the fabrication. Each cell of the structure has four waveguides that allow the excited HOMs to propagate and dissipate into rf absorbers. With respect to the design reported in Refs. $[13,14]$ based on broadband individual $\mathrm{SiC}$ rf absorbers, our design is based on $\mathrm{SiC}$ 


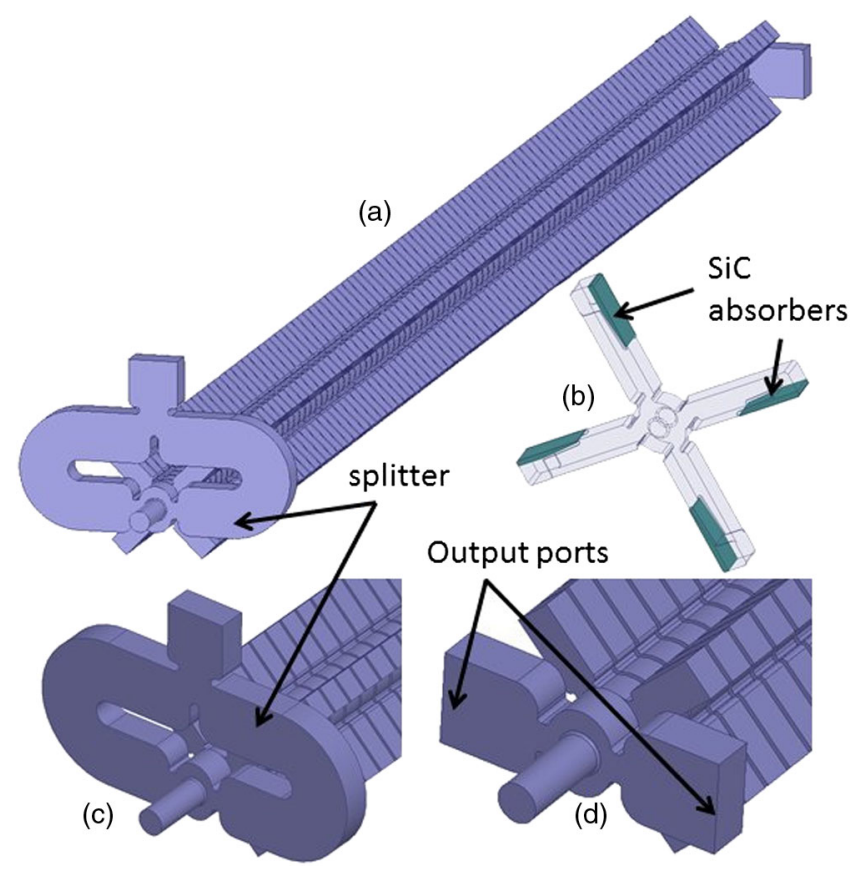

FIG. 1. Sketch of the $C$-band cavity: (a) whole structure; (b) accelerating cell detail with highlighted $\mathrm{SiC}$ absorbers; (c) racetrack input coupler with integrated splitter; (d) racetrack output coupler.

"tiles" with a very simple shape (rectangular type), inserted and fixed in modules of 12 cells. Such a design also has another advantage from the vacuum conductance point of view, because all cells have a hole form cell to cell in the proximity of the $\mathrm{SiC}$ absorber that allows them to have a high vacuum conductance in the proximity of the $\mathrm{SiC}$

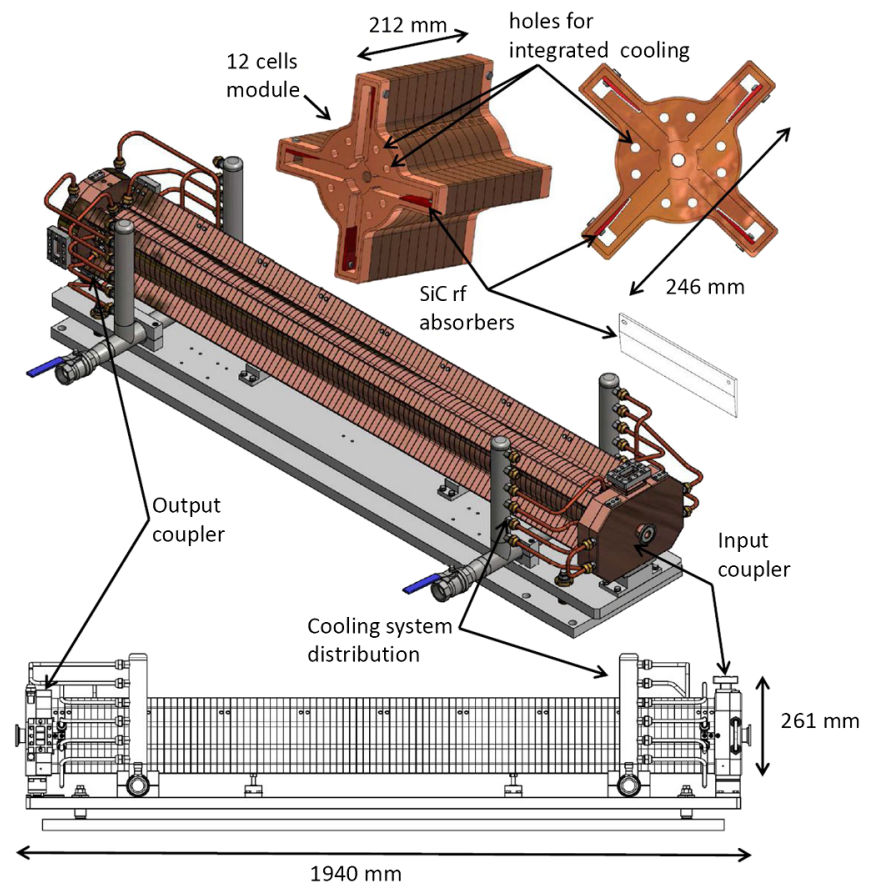

FIG. 2. Mechanical drawings of the $C$-band structures.
TABLE I. Main parameters of the ELI-NP accelerating structures.

\begin{tabular}{lc}
\hline \hline Parameter & Value \\
\hline Working frequency $\left(f_{\text {rf }}\right)$ & $5.712 \mathrm{GHz}$ \\
Cell phase advance & $2 \pi / 3$ \\
Number of cells & 102 \\
Structure length & $1.8 \mathrm{~m}$ \\
Iris aperture radius & $6.8-5.78 \mathrm{~mm}$ \\
Repetition rate & $100 \mathrm{~Hz}$ \\
Average quality factor & 8850 \\
Average accelerating field & $33 \mathrm{MV} / \mathrm{m}$ \\
Shunt impedance & $67-74 \mathrm{M} \Omega / \mathrm{m}$ \\
Group velocity $\left(v_{g} / c\right)$ & $0.025-0.015$ \\
Filling time & $313 \mathrm{~ns}$ \\
rf input power $\left(P_{\text {in }}\right)$ & $40 \mathrm{MW}$ \\
Output power $\left(P_{\text {out }}\right)$ & $0.29 \mathrm{P}_{\text {in }}$ \\
Pulse duration for beam $\left(\tau_{\text {beam }}\right)$ & $<512 \mathrm{~ns}$ \\
Pulsed heating (input coupler) & $<21^{\circ} \mathrm{C}$ \\
Average wall-loss power & $2.3 \mathrm{~kW}$ \\
Working temperature & $30^{\circ} \mathrm{C}$ \\
\hline \hline
\end{tabular}

absorbers themselves (Fig. 2). This allows one to increase the effective pumping speed along the structure. The input and output couplers have two symmetric ports and race track cell geometries to minimize the dipole and quadrupole field components introduced by the coupling holes. A splitter integrated in the input coupler allows a symmetric rf powering of the cavity, while the output one has two symmetric output ports connected to the two rf loads. In Sec. II, the fabrication process and the brazing procedure of the structures will be illustrated. In Sec. III, the results of the low-power rf measurements in terms of scattering parameters, field profile, effectiveness of the damping system and tuning will be discussed. Finally, in Sec. IV, the results of the high power rf tests will be reported.

\section{FABRICATION PROCESS}

The mechanical drawings of the structure are shown in Fig. 2. Each structure is composed of ten modules: the input and output coupler (with the two adjacent cells) and eight 12-cell modules. This module-based assembly is for realizing better control of the brazing process. The modules, in fact, are fabricated, brazed, and vacuum tested separately and are finally brazed all together after the insertion of the SiC absorbers. As illustrated in Ref. [10], the geometry of the $\mathrm{SiC}$ absorbers has been strongly simplified with respect to the CLIC structures, and they resulted as four tiles inserted in each module. This simplifies the absorber fabrication procedure and reduces the overall production cost. Moreover, another advantage of the proposed solution is the pumping of the $\mathrm{SiC}$ absorber regions through the opening holes in the region of the absorbers themselves, that strongly reduce vacuum 


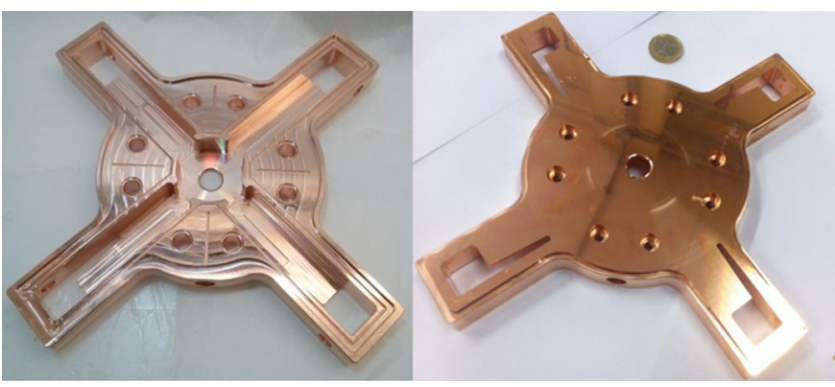

FIG. 3. Pictures of a fabricated cell.

impedances, allowing a much better pumping of the structure. The fabrication of all the copper components has been done by one company [15].

The manufacturing of the cells proceeded in several steps. After a rough machining, the cells underwent a stress-relieving treatment in a vacuum furnace at $500^{\circ} \mathrm{C}$ for one hour. Such treatment allowed the relief of any internal stress causing unwanted deformations due to machining and/or successive brazing procedures. Then, they have been milled (with the Vertical Work Center Hass VF 3 SS), while the final machining of the irises has been performed by means of the ultraprecise lathe Schaublin $225 \mathrm{TM}-\mathrm{CNC}$, that guarantees a precision of $\pm 5 \mu \mathrm{m}$ and a surface roughness lower than $50 \mathrm{~nm}$. Moreover, four deformation tuners of the type already implemented and illustrated in Ref. [16] and eight cooling pipes have been obtained, by milling, in each cell. Pictures of machined cells are given in Fig. 3. Each cell has been checked with an automatic 3D measuring machine to verify all internal dimensions.

The manufacturing of the input and output couplers followed a similar procedure. They have been roughly machined (with the Vertical Work Center Doosan DNM $750 \mathrm{~L}$ ) and underwent a stress-relieving treatment in the vacuum furnace. The final machining has been done with the Vertical Work Center Mikron UCP 600 Vario, with a precision on the internal dimensions lower than $\pm 10 \mu \mathrm{m}$ and with a surface roughness $<150 \mathrm{~nm}$. Pictures of the couplers during the fabrication process are shown in Fig. 4. Then, all components have been cleaned in several steps: using neutral soaps, weak acid (citric), and Almeco 19 and $\mathrm{NGL}^{1}$ soap in an ultrasound bath.

The 12-cell modules have been brazed at $850^{\circ} \mathrm{C}$ with Palcusil 10 alloy [17]. All external module dimensions have been verified with a 3D measuring machine (before and after the brazing process) and have been vacuum tested with a sensitivity lower than $5 \times 10^{-10} \mathrm{mbar} 1 / \mathrm{s}$. The $\mathrm{SiC}$ absorber (in Ekasic-P [18]) tiles have been machined with a precision of $\pm 20 \mu \mathrm{m}$ and a roughness $<500 \mathrm{~nm}$ and have been outgassed in a vacuum oven at $900^{\circ} \mathrm{C}$ for one hour. Then, they have been inserted in the cells and fixed with special screws that allow one to take into account the

\footnotetext{
${ }^{1}$ https://www.ngl-group.com/en/.
}

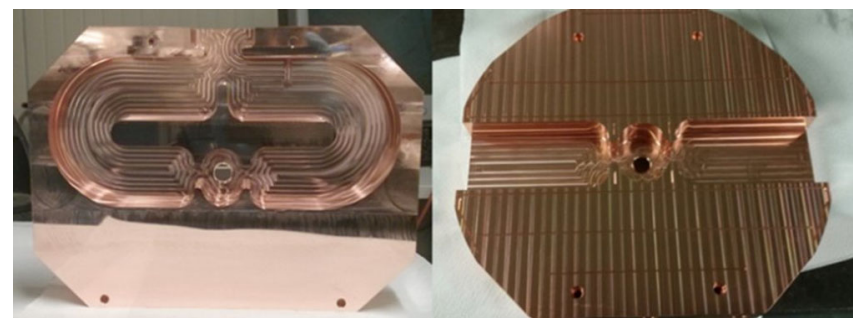

FIG. 4. Machined input (left) and output (right) coupler components.

different thermal expansion of copper and $\mathrm{SiC}$ absorbers during the following brazing phases. A picture of a brazed 12-cell module is shown in Fig. 5, with a detail of the $\mathrm{SiC}$ absorber fixed inside the module itself.

The two couplers have been brazed similarly. Pictures of the input and output couplers are given in Fig. 6 with details of the rf absorbers on the two cells brazed together with the couplers themselves.

The last two brazing steps have been performed in the INFN-LNL Legnaro laboratories, where a large vacuum furnace is available. The INFN-LNL vacuum furnace allows brazing $1.5 \mathrm{~m}$ long structures. For longer structures (up to about $2 \mathrm{~m}$, as in our case), one has to insert a notheated extension (i.e., extension without heating resistors). In order to avoid brazing junctions (between modules) to be

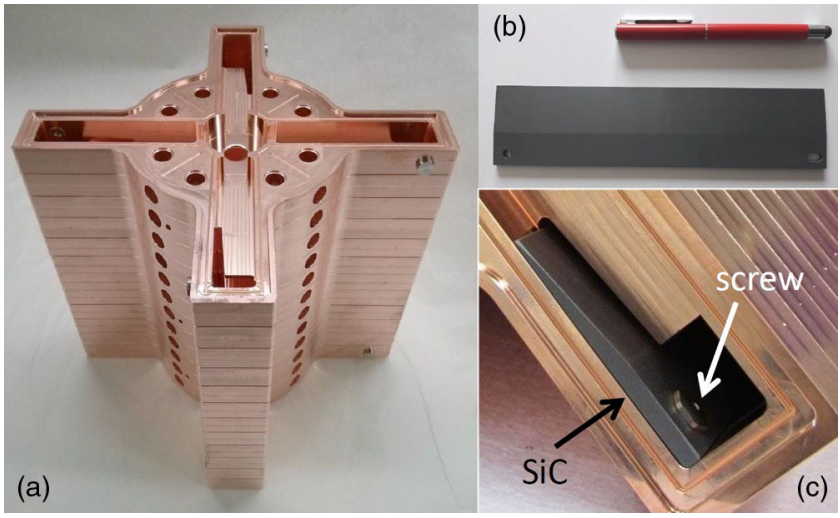

FIG. 5. Brazed 12-cell module (a); SiC absorber (b); detail of the $\mathrm{SiC}$ absorber fixed inside the module (c).

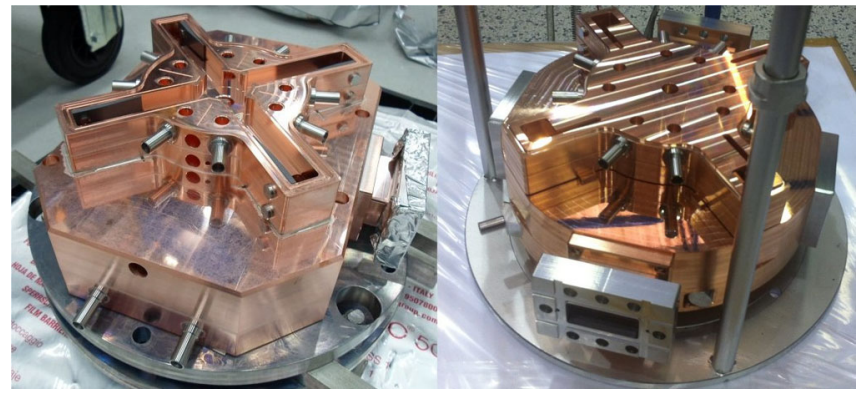

FIG. 6. Brazed input coupler (left) and brazed output coupler (right). 


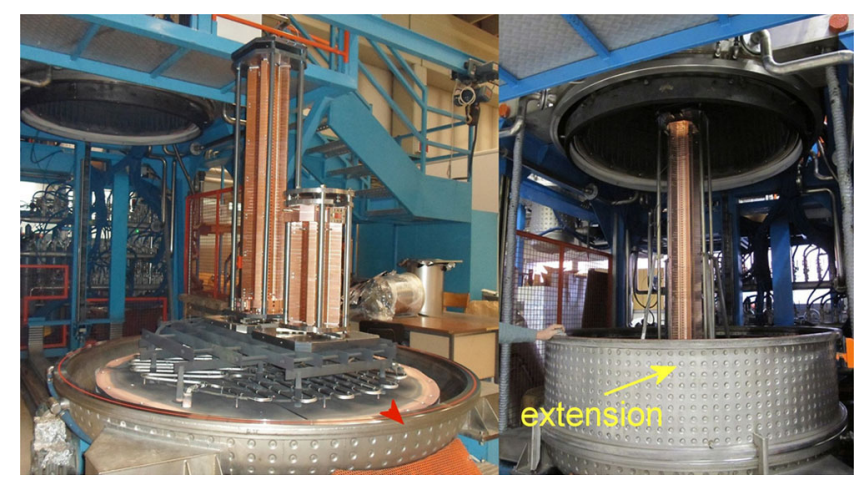

FIG. 7. Two half structures before brazing (left); full assembled structure in the INFN-LNL oven with the not-heated extension (right).

in the region of the oven "close" to the not-heated extension, the brazing has been divided in two steps: the brazing of two "short" subassemblies (in the oven without the not-heated extension) and the final brazing of the whole structure (in the oven with the extension). Pictures of the two sub-assemblies are given in Fig. 7: the first subassembly was composed of six modules and the output coupler, while the second one was made of the remaining two modules with the input coupler. Palcusil 5 alloy at $810^{\circ} \mathrm{C}$ has been used in this brazing phase. In the last oven cycle, the two sub-assemblies have been joined by the Cusil alloy at $780^{\circ} \mathrm{C}$. The picture of the full assembled structure in the INFN-LNL oven is given in Fig. 7.

A leak test (with a sensitivity lower than $5 \times 10^{-10} \mathrm{mbar} \mathrm{l} / \mathrm{s}$ ) has been done after the final brazing and has been repeated after a moderate bakeout at $100^{\circ}$ for $12 \mathrm{~h}$. This bakeout has been done just to speed up the outgassing of the surfaces reaching faster the desired pressure. Finally, the structure reached a pressure of a few $10^{-9}$ mbar with one ion pump connected on one side. We also performed a residual gas analysis (RGA), and the result is given in Fig. 8. From the plot, it is quite easy to

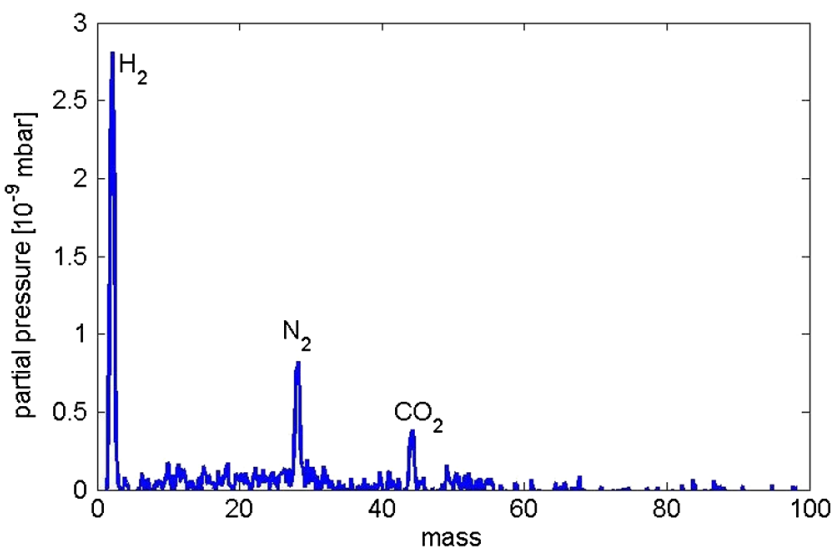

FIG. 8. RGA scan of the structure after brazing.

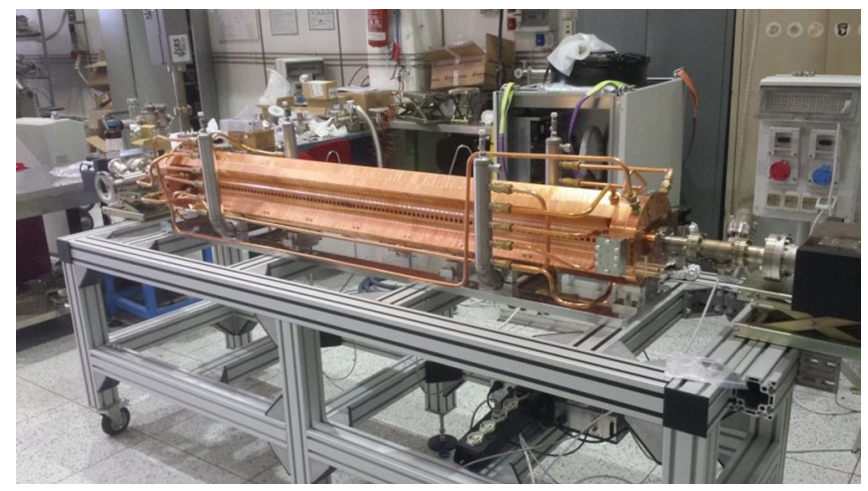

FIG. 9. $\quad C$-band structure with the cooling system installed and under pumping.

note that there is no peak at the mass of water. This was due to the careful dry nitrogen venting and flushing during all installation phases. The cooling channel distribution has then been implemented, and the picture of the final structure under vacuum pumping is shown in Fig. 9.

\section{LOW-POWER TESTS AND TUNING RESULTS}

The picture of the structure under low-power rf tests is shown in Fig. 10. The scattering parameters have been measured at the input and output ports with a network analyzer (Rohde \& Schwarz ZVB 20). The magnitude of the accelerating field and the phase advance per cell have been measured with the bead pull technique using a metallic sphere fixed on a nylon wire [Fig. 10(c)]. The electric field and the phase advance per cell measured before and after the tuning procedure are shown in Figs. 11 and 12, for the first structure prototype and the sixth

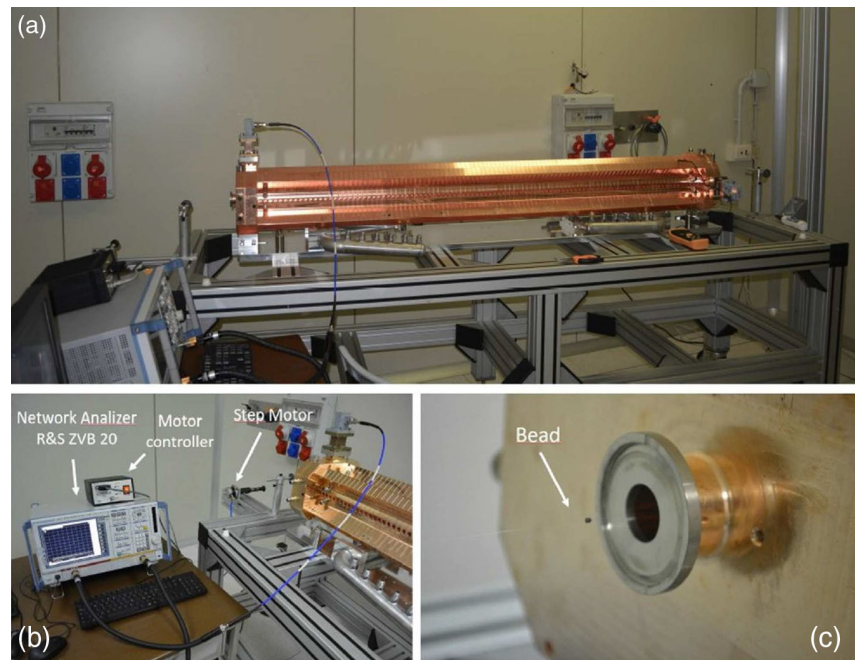

FIG. 10. Pictures of the structure under low-power rf tests and tuning: (a) cavity under test; (b) detail of the network analyzer and motor control; (c) metallic bead used for the field measurements. 
(a)
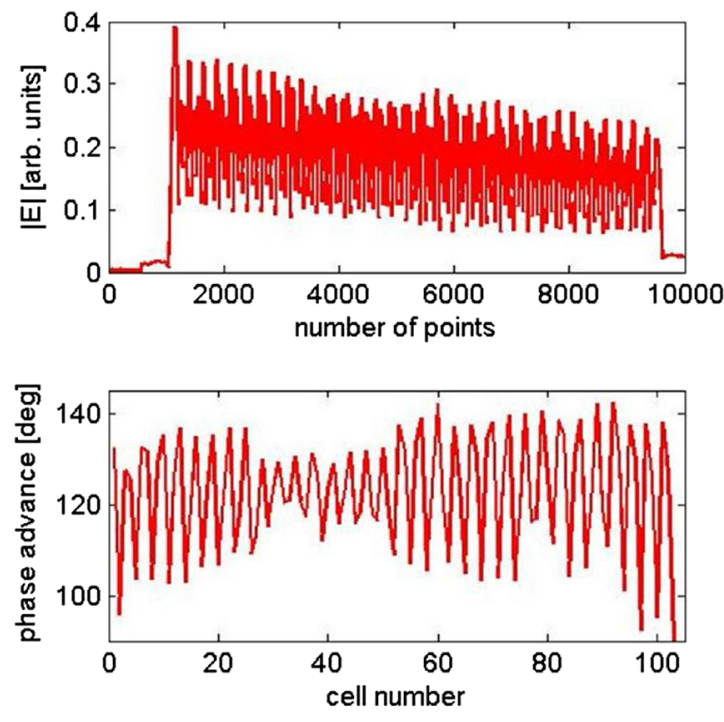

(b)
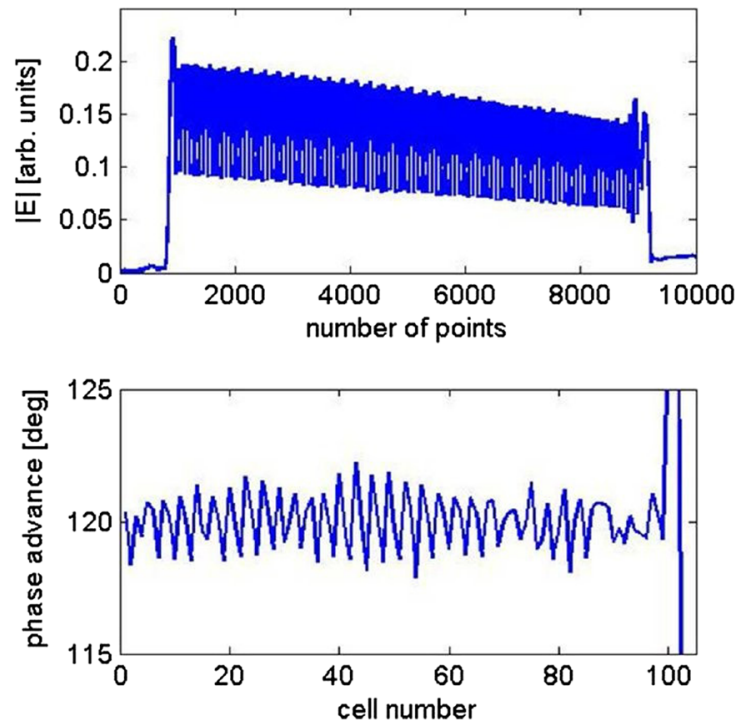

FIG. 11. Magnitude (upper plots) and phase advance per cell (bottom plots) of the prototype accelerating structure before (a) and after (b) tuning.

fabricated structure, respectively. ${ }^{2}$ The tuning has been done acting on four small deformation tuners using the same procedure already illustrated and adopted in Refs. [7,16] and following the procedure illustrated in Ref. [16]. As extensively discussed in Ref. [19] and briefly summarized in the Appendix, the requirements in terms of cell-to-cell $\left(\Delta \phi_{n}\right)$ and cumulative $\left(\phi_{\text {cum }}\right)$ phase advances to have a reduction of the total accelerating voltage experienced by the particles (with respect to the nominal one) lower than $0.2 \%$ are $-2^{\circ} \leq \Delta \phi \leq+2^{\circ}$ and $-5^{\circ} \leq \phi_{\text {cum }} \leq+5^{\circ}$. A reflection coefficient $\left(S_{11}\right)$ at the input port lower than $<-20 \mathrm{~dB}$ assures also to have a reduction of the total accelerating voltage due to the input coupler reflection lower than $0.5 \%$. The measurements and tuning have been done at an equivalent frequency, taking into account the different structure temperature $\left(22^{\circ} \mathrm{C}\right.$ instead of $30^{\circ} \mathrm{C}$ ), the presence of air, and the shift due to the nylon wire as described in Ref. [19]. In the first prototype, the distortion of the accelerating field in the last

\footnotetext{
${ }^{2}$ In the field measurement, it is possible to note that the measured field immediately after the output coupler does not go to zero, as expected. This is due to the fact that the field measurement in each point is obtained by subtracting the perturbed $S_{11}$ (with the bead in) and the unperturbed one (bead out). The unperturbed $S_{11}$ is that measured when the bead is out of the structure (i.e., when it is before the input coupler or above the output one). In both conditions, the nylon wire is inserted in the structure, and, obviously, the two pieces of nylon wires are different in the two cases (since they are before or above the bead). In the presented measurements, we considered as unperturbed $S_{11}$ the case in which the bead was out of the structure immediately before the input coupler. The small pedestal at the end of the structure (after the output coupler) is due to the slightly different unperturbed $S_{11}$ when the bead was on the other side.
}

three cells (and the corresponding oscillations on the cellto-cell phase advances above the mentioned range) have been introduced on purpose during the tuning phase, in order to compensate for the reflections coming from the output coupler (probably due to a deformation occurred during the brazing process). The associated decrease in the overall structure efficiency is still below 2\%. For the realization of the other structures, the radius of the output coupler has been increased about $50 \mu \mathrm{m}$ to compensate for this distortion. This value has been calculated by simulating the structure with the electromagnetic code used for the design (ANSYS-ELECTRONICS [20]) and reproducing the obtained rf measurement results. As a result, the flatness and phase advances on the lasts cells after tuning were within specifications for all other structures, as visible from Fig. 12. From the plots, it is possible to remark that (excluding the last cells of the prototype) the final field smoothness is below $1 \%$, while the final phase advance per cell is the nominal one within $\pm 2^{\circ}$. The cumulative phase advance per cell has been also measured, and it stays within $\pm 5^{\circ}$. The reflection coefficient at the input port of the sixth structure before and after the tuning is given in Fig. 13(a), as an example. At the working frequency and after tuning, it is lower than $-25 \mathrm{~dB}$. The measured transmission coefficient between the input port and one of the output ones is shown in Fig. 13(b). The transmission coefficient from the input coupler to one of the output coupler ports is about $-8.5 \mathrm{~dB}$; this is in good agreement with the total structure attenuation as given in Ref. [10] and reported in Table I.

For the sake of completeness, we also report here the lowpower rf tests performed on a single 12-cell module already illustrated in a previously published paper [10]. The results, given in Fig. 14, show the transmission coefficient between 
(a)
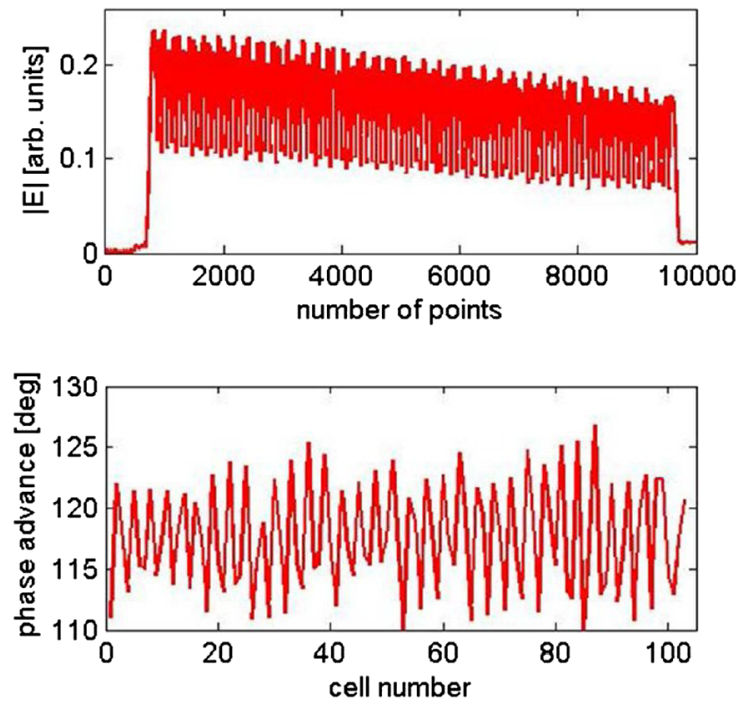

(b)
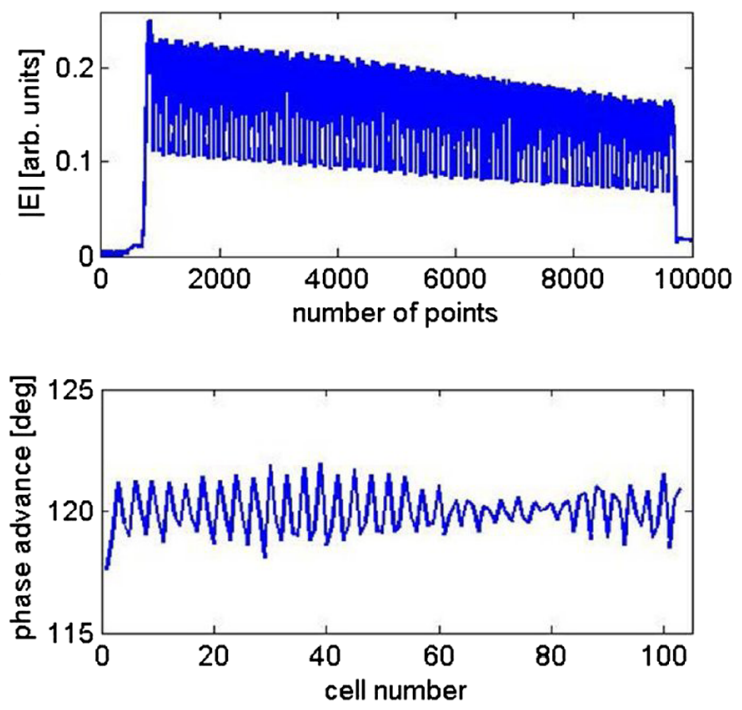

FIG. 12. Magnitude (upper plots) and phase advance per cell (bottom plots) of the sixth accelerating structure before (a) and after (b) tuning.

(a)

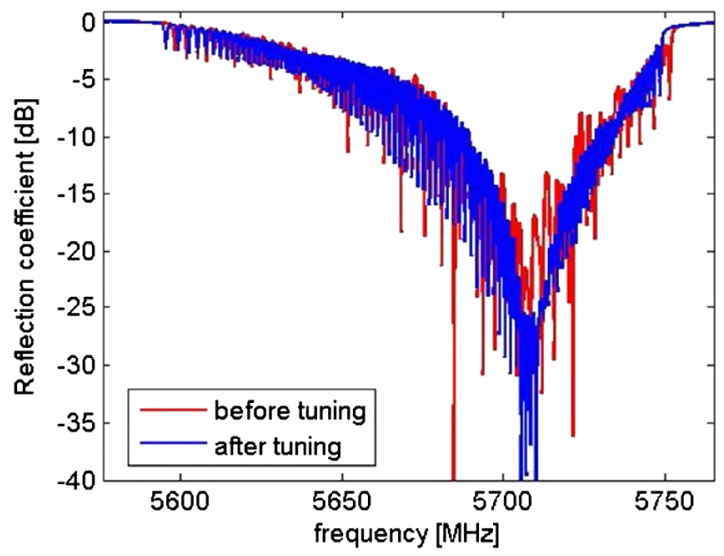

(b)

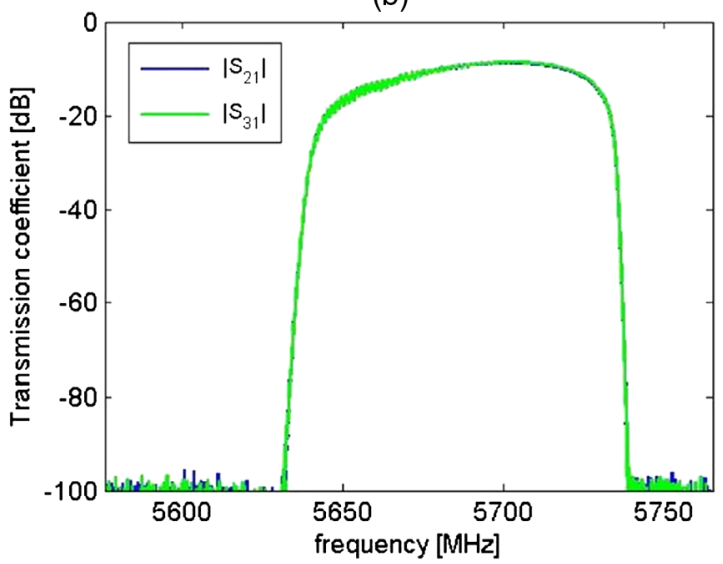

FIG. 13. Reflection coefficient at the input port before and after tuning for the sixth tuned structure (a); measured transmission coefficient between the input port and the output ones after tuning (b).
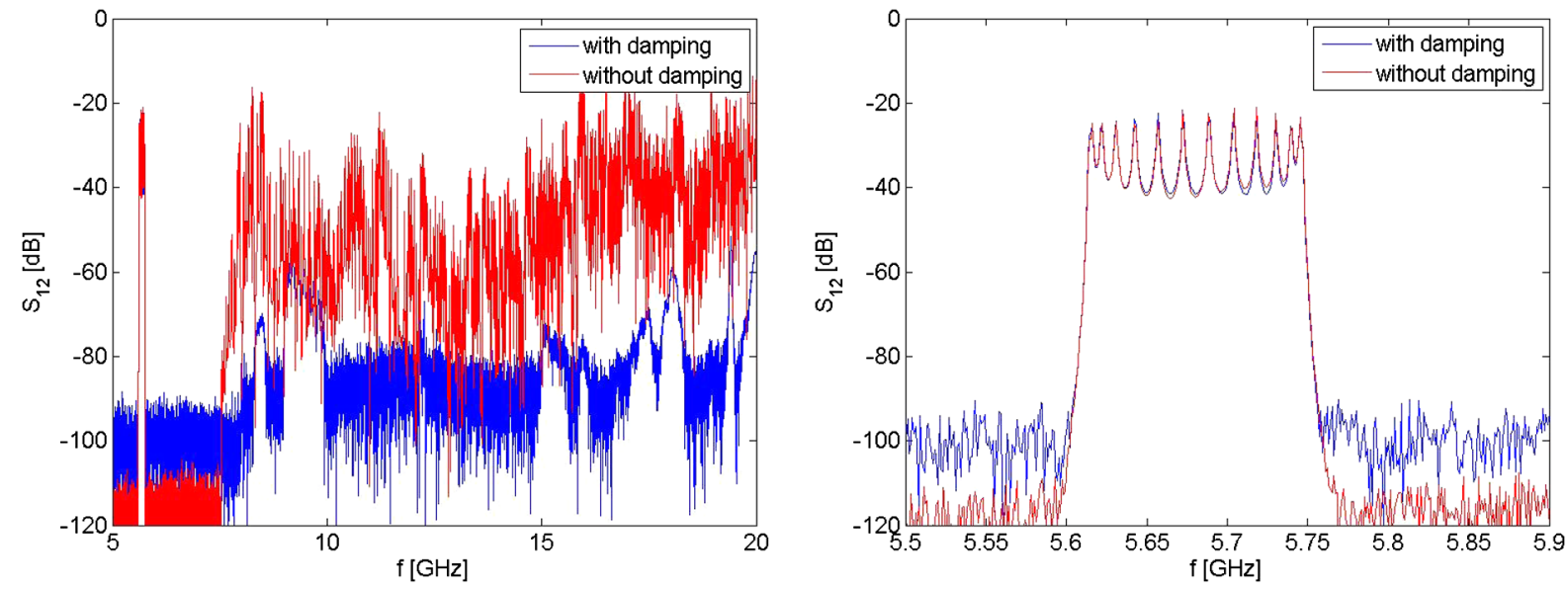

FIG. 14. Transmission coefficient between two antennas coupled to a 12-cell module with and without the absorbers (left); detail of the transmission coefficient corresponding to the bandwidth of the accelerating mode (right) [10]. 
two antennas coupled with the 12-cell structure with and without the rf $\mathrm{SiC}$ absorbers. ${ }^{3}$ The measurements show the effectiveness of the $\mathrm{SiC}$ absorbers, since the HOMs disappear after the insertion of the absorber themselves. The remaining modes with quality factors of a few hundred are TE-like modes that have a negligible transverse impedance. On the other hand, the accelerating mode band, the lowest passband, is unperturbed as clearly visible in Fig. 14 (right plot).

\section{HIGH POWER TEST RESULTS}

The first prototype structure has also been tested at high power at the Bonn University under the responsibility of a private company [21]. The picture of the structure during such test is given in Fig. 15(a), and the experimental setup is schematically presented in Fig. 15(b). The $C$-band structure installation started on 17 February, 2015. The conditioning of the structure started on 18 March, 2015 and ended on 23 April, 2015. The power source was the one foreseen for the ELI-NP project: a Scandinova [22] rf unit based on solid state modulator K2-2 adapted for 50 MW $C$-band Canon klystron E37212. ${ }^{4}$ In the high power installation, we used the directional couplers $\mathrm{DC}_{1}$ and $\mathrm{DC}_{2}$ to measure the input and reflected powers and the transmitted one. Moreover, the ceramic window ${ }^{5}$ before the input coupler has been inserted to avoid the klystron window being directly exposed to the vacuum of the cavity both during installation and then under conditioning. It has been a "safety measure" to protect the klystron itself, because we did not know either the vacuum level in the cavity under high power or the effects of the $\mathrm{SiC}$ absorbers outgassing.

The aim of the rf conditioning was to reach the nominal parameters in terms of input power at the input coupler (40 MW), repetition rate $(100 \mathrm{~Hz})$, and pulse length ( $820 \mathrm{~ns}$, corresponding to one filling time plus beam time for multibunch operation). The forward and reflected power signals at the input and output couplers were measured using diode peak signal detector readout with oscilloscopes. The diodes have been calibrated in the laboratory using a CW rf source monitored by a power meter and a 12-bit oscilloscope to read the diode output voltage level.

\footnotetext{
${ }^{3}$ The two different noise levels, with and without the absorbers, could be generated by several causes. One could be the fact that, in order to mount and dismount the absorbers, we had to open the two plates closing the modules, dismount the absorbers, and close again the structure. In this condition, the contact between the two plates probably changed and/or the connection was not exactly the same, giving this different noise floor.

${ }^{4}$ The main klystron and modulator parameters are: rf frequency $5712 \mathrm{MHz}$, maximum rf peak power $50 \mathrm{MW}$, maximum rf average power $5 \mathrm{~kW}$, operational voltage range $0-350 \mathrm{kV}$, operational current range $0-317 \mathrm{~A}$, repetition rate range $0-100 \mathrm{~Hz}$, maximum rf pulse length $1 \mu$ s, and amplitude stability $< \pm 0.005 \%$.

${ }^{5}$ The insertion loss of the window is below $0.05 \mathrm{~dB}$, and the VSWR is below 1.1.
}

The directional couplers have been calibrated in the laboratory using waveguide-coaxial transitions and a network analyzer. The power measurements agreed within 5\% with the calibration of the Canon company, in terms of the expected output power as a function of the klystron high voltage in saturation conditions (klystron datasheet).

The voltage signals were recorded and converted into power levels by means of a LabVIEW GUI running on a PC in the control room. The klystron power, repetition rate, and pulse length were progressively increased while the current of three ion pumps (connected to the input and output waveguides) and the rf signals from pickups were monitored. The conditioning procedure was semiautomatic, and the switch off on the modulator high voltage could be caused by: (a) operators; (b) ion pump current exceeding a threshold corresponding to a pressure of $1 \times 10^{-7}$ mbar; or (c) reflected power to the klystron exceeding $10 \%$ of the input one. The input, transmitted, and reflected power signals are given, as an example, in Fig. 16 in the case of a $30 \mathrm{MW}$ input power $0.35 \mu$ s pulse length. The signals are not to scale, since we used different attenuations for the diodes, but the measured value of the transmitted and reflected power were in perfect agreement with the lowpower measurements in terms of attenuation and reflection.

The rf conditioning lasted about 190 h. In Fig. 17, the behaviors of the rf pulse length, input power, and repetition rate as a function of time are reported. During the conditioning, we initially kept constant the repetition rate at $50 \mathrm{~Hz}$ and the rf pulse length at around $100 \mathrm{~ns}$, and we progressively increased the input power. This allowed us to keep under control the vacuum pressure and to make a first conditioning of the structure and waveguide components (including the ceramic window, loads, directional couplers, and waveguides). After about $40 \mathrm{~h}$, we increased the repetition rate to $100 \mathrm{~Hz}$. Then we progressively increased (in steps of about 100-200 ns) the pulse length up to the nominal value of 820 ns. For each pulse length step, we make a conditioning, increasing the power level up to the maximum power of $40 \mathrm{MW}$. In some cases, to recover the vacuum level due to breakdowns, we decreased the repetition rate and input power for a few minutes. Before increasing the rf pulse length, we waited a few hours (1$2 \mathrm{~h}$ ) at full power, full repetition rate, and stable operation in terms of breakdown rate (BDR) [a BDR less than 2-3 per hour means a BDR less than $10^{-5}$ breakdown per pulse (bpp)]. The vacuum pressure was constantly measured by the ion pump currents during the whole process. Its baseline at full power was of the order of $1 \times 10^{-8}$ mbar and always maintained a decreasing trend. The vacuum pressure, measured at the entrance and at the end of the structure from ion pumps 2 and 3 during conditioning, is given in Fig. 18. During the test, the vacuum level of ion pump 1 was useful to discriminate discharges happening in the waveguide from those in the structure. The vacuum peaks in Fig. 18 correspond to breakdown phenomena at the 
(a)

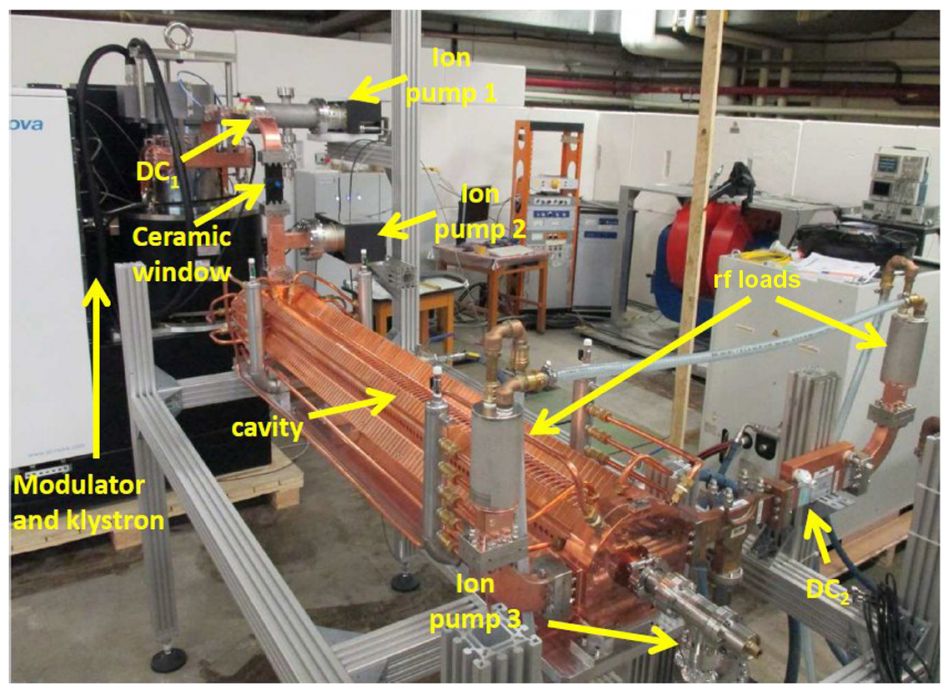

(b)

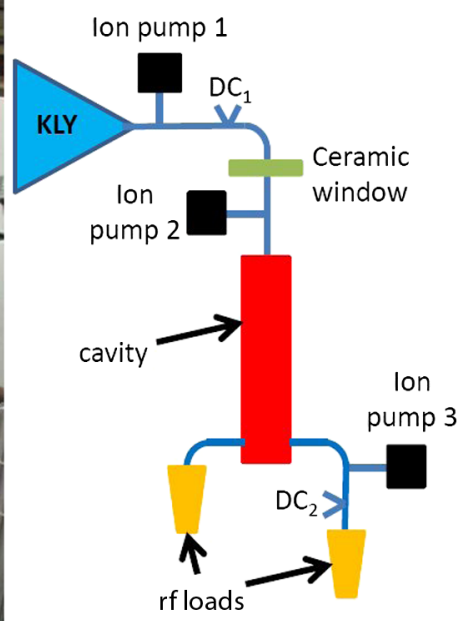

FIG. 15. $\quad C$-band structure under a high power test (a); scheme of the high power test layout (b).

entrance and at the end of the structure and have been used to calculate the number of breakdowns and the final BDR. In the calculation, we have considered as a "vacuum peak" any relative jump in the vacuum pressure. The results are reported in Figs. 19 and 20 and clearly show the larger number of breakdowns in the initial part of the structure related to the slightly larger electric field. For the BDR, we have considered a moving time window of about $10^{4} \mathrm{~s}$. The breakdown rate, at the end of the conditioning, was of the order of a few $10^{-6} \mathrm{bpp} / \mathrm{m}$.

It is important to remark that during the test, for questions related to the delivery of the structure and timeline of the

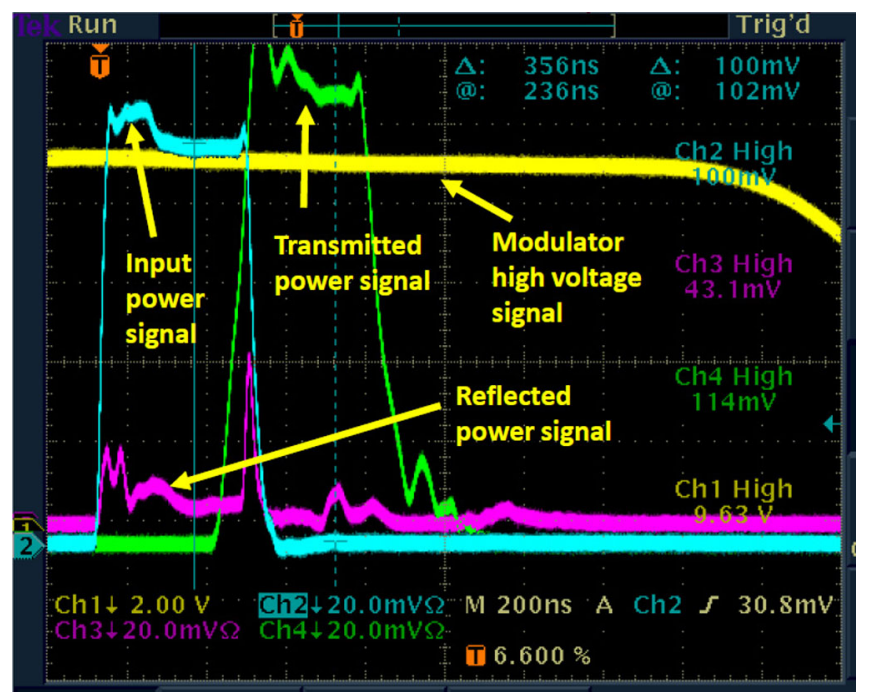

FIG. 16. Input, transmitted, and reflected power signals in the case of $30 \mathrm{MW}$ input power and $0.35 \mu$ s pulse length. The signals are not to scale, since we used different attenuations for the diodes. project, the structure has not been fully conditioned. Typically, hundreds of million pulses are required for a full conditioning of the structures to reach the ultimate BDR [23-25], that means, at a repetition rate of $100 \mathrm{~Hz}$, several hundreds of hours. For this reason, the final BDR is expected to be well below the level we reached $\left(10^{-6} \mathrm{bpp} / \mathrm{m}\right)$. Nevertheless, even in this short time, we reached a BDR comparable with that obtained, as an example, in the first
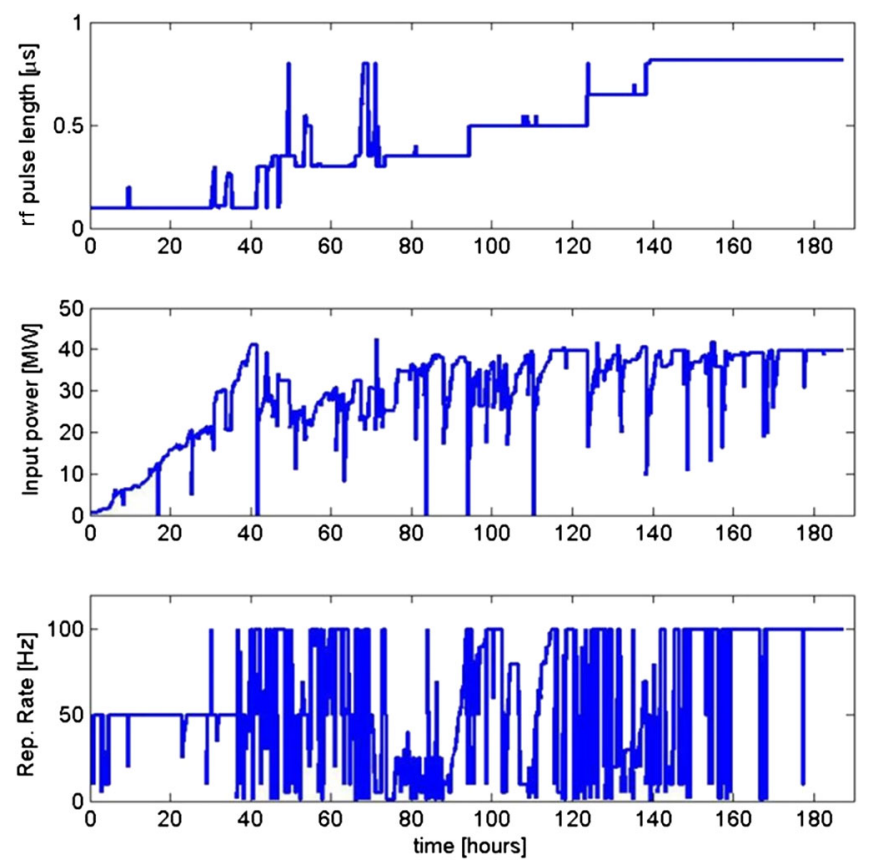

FIG. 17. Behavior of the rf pulse length, input power, and repetition rate as a function of time during the high power test of the $C$-band structure. 

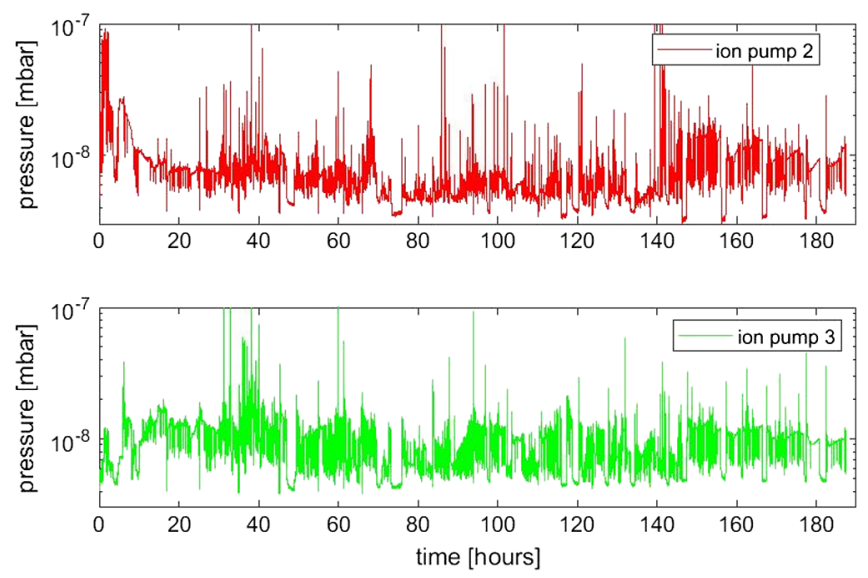

FIG. 18. Vacuum pressure measured from ion pumps 2 and 3 as a function of the conditioning time.

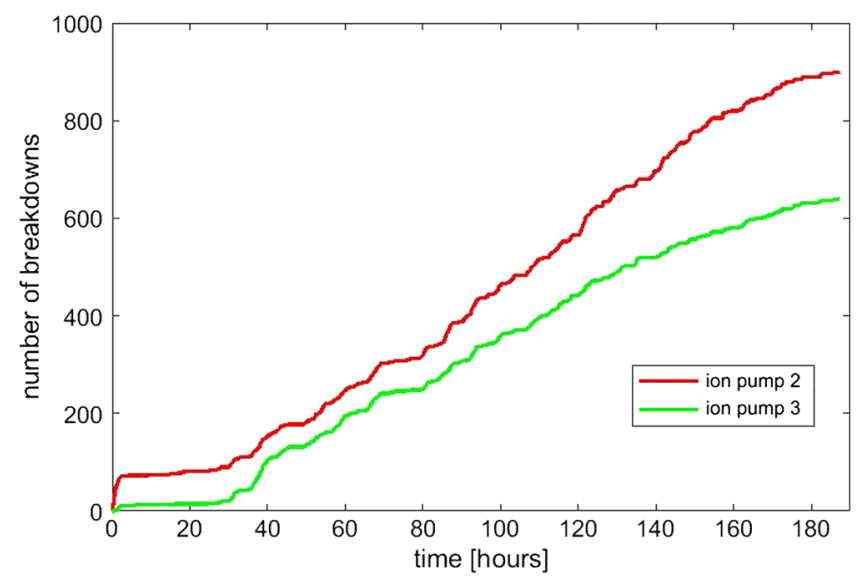

FIG. 19. Number of cumulated breakdowns calculated by peaks on a vacuum measured by ion pumps 2 and 3 .

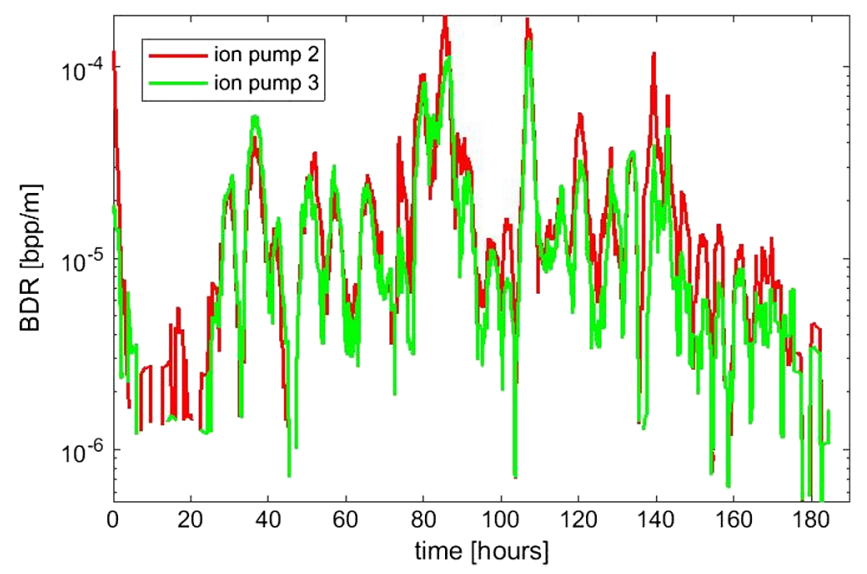

FIG. 20. Calculated BDR as measured by peaks on a vacuum measured by ion pumps 2 and 3 and considering a moving time window of about $10^{4} \mathrm{~s}$. tested $C$-band structures at PSI [26] or measured at Spring-8 [6] with comparable gradients but shorter pulses than in our case. The SPARC_LAB ones [7] reached a higher BDR, because the structures have been conditioned with a much lower number of total integrated pulses.

It is also important to underline that we performed a "vacuum conditioning" of the structure, but, at a very high gradient, it is also possible to have breakdown activity even without vacuum activity. This activity can be detectable with more complex systems that allow one to detect not only the vacuum signals but also the rf and dark current signals in real time mode, allowing feedback on the input power. As pointed out, these automatic detection systems were not implemented in our case. Nevertheless, at the reached level of power and surface fields, we did not observe any significant breakdown activity, monitored by the rf signals on the oscilloscope connected to the diodes in "infinite persistence" mode, without vacuum activity. This, of course, does not exclude the presence of a residual breakdown activity detectable with more sensitive systems.

\section{CONCLUSIONS}

In this paper, the fabrication procedure and the low and high power rf tests of the HOM damped $C$-band structures for the ELI-NP gamma beam system have been illustrated. The structures are $1.8 \mathrm{~m}$ long TW cavities and integrate an HOM damping system based on waveguides coupled to each cell. Each structure consisted of modules fabricated, brazed, and tested separately and then brazed all together after the insertion of the $\mathrm{SiC}$ absorbers. The geometry of the $\mathrm{SiC}$ absorbers has been strongly simplified, and they resulted as four tiles inserted in each module. This simplified the absorber fabrication procedure, reducing the overall production cost. The cavities have been tuned by small deformation tuners and reached the field smoothness of $<1 \%$, the phase advances per cell with an error lower than $\pm 2^{\circ}$, and the cumulative phase advance per cell within $\pm 5^{\circ}$. The first full-scale prototype structure has been successfully tested at high power, reaching, in about $190 \mathrm{~h}$ of $\mathrm{rf}$ conditioning, the nominal parameters in terms of gradient $(33 \mathrm{MV} / \mathrm{m})$, repetition rate $(100 \mathrm{~Hz})$, and pulse length (820 ns).

\section{ACKNOWLEDGMENTS}

We thank F. Pellegrino, V. Pettinacci, and M. Magi for the technical support on prototype cavity installation, P. Chimenti and S. Bini for their technical support on cavity vacuum tests and A. Falone for helpfull suggestions and management support. We would like also to acknowledge the COMEB [15] and RI [21] Companies for their technical support in the realization of the structures and in the high power test of the prototype, respectively. 
(a)

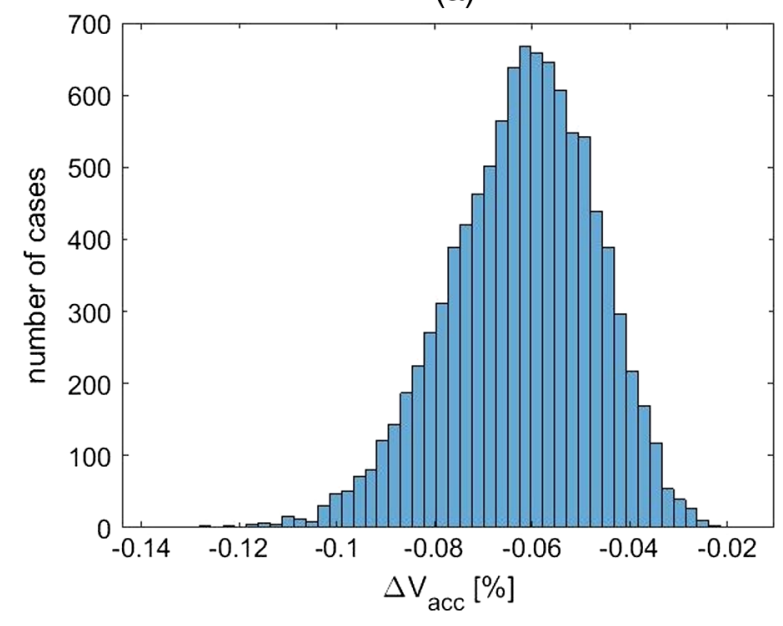

(b)

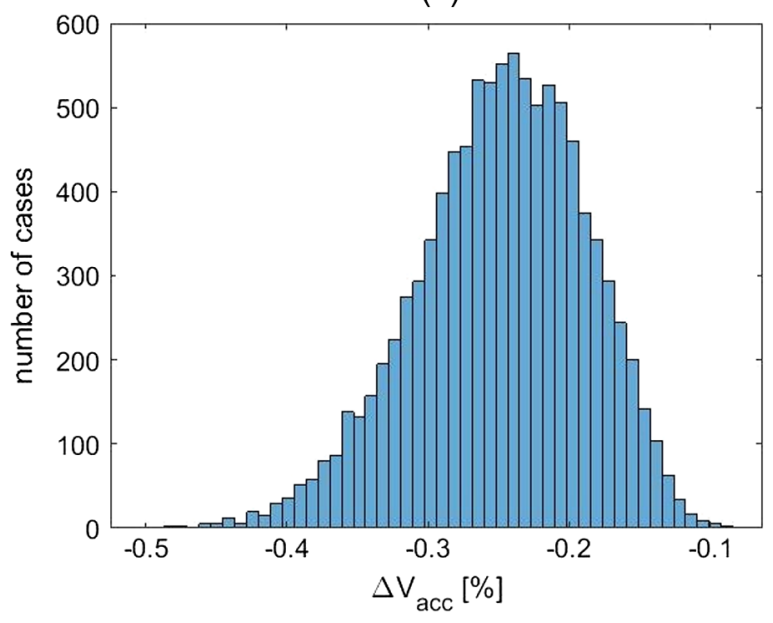

FIG. 21. Distribution of the reduction in the average accelerating voltage calculated considering $10^{4}$ random cases: (a) maximum cellto-cell phase advance deviation of $\Delta \phi_{n}= \pm 2^{\circ}$ and maximum cumulative phase advance error along the structure $\left(\phi_{\text {cum }}\right)$ within $\pm 5^{\circ}$ (mean value $-0.07 \%$, standard deviation $-0.015 \%$, minimum value $-0.15 \%$ ); (b) maximum cell-to-cell phase advance deviation of $\Delta \phi_{n}= \pm 4^{\circ}$ and maximum cumulative phase advance error along the structure $\left(\phi_{\text {cum }}\right)$ within $\pm 10^{\circ}$ (mean value $-0.2485 \%$, standard deviation $-0.06 \%$, minimum value $-0.5 \%$ ).

\section{APPENDIX: ANALYSIS OF THE CELL-TO-CELL AND CUMULATIVE PHASE ADVANCE ERRORS ON ACCELERATING STRUCTURE PERFORMANCES}

As reported, e.g., in Refs. [27,28], the energy gain $(\Delta W)$ of an ultrarelativistic particle of charge $q$ traveling across a TW structure can be expressed as

$$
\begin{aligned}
\Delta W & =q \cdot V_{\mathrm{acc}} \\
& =q \int_{0}^{L} E(z) \cdot \cos \left[\omega_{\mathrm{rf}} \frac{z}{c}-\int_{0}^{z} k\left(z^{\prime}\right) d z^{\prime}+\varphi_{\mathrm{inj}}\right] d z,
\end{aligned}
$$

where $E(z)$ is the accelerating field along the axis of the structures, $L$ is the structure length, $\omega_{\mathrm{rf}}$ is the angular $\mathrm{rf}$ frequency of the accelerating field, $k(z)$ is the wave number, and $\varphi_{\text {inj }}$ is the electron injection phase. Converting the previous equation in a discrete domain, we obtain

$$
V_{\mathrm{acc}}=\sum_{i=1}^{N} E_{i} D \cdot \cos \left(\omega_{\mathrm{rf}} \frac{i \cdot D}{c}-\sum_{n=1}^{i} \phi_{n}+\varphi_{\mathrm{inj}}\right),
$$

where $D$ is the single-cell length and $\phi_{n}$ is the phase advance per cell of the $n$ cell defined by $\phi_{n}=$ $\int_{(n-1) D}^{n D} k\left(z^{\prime}\right) d z^{\prime}$. Recognizing that $\omega_{\mathrm{rf}} \frac{D}{c}=\phi_{\text {nom }}$ is the nominal phase advance per cell $\left(\phi_{\text {nom }}=2 \pi / 3\right.$ in the ideal case of the ELI-NP structures), we can easily obtain

$$
\begin{aligned}
V_{\mathrm{acc}} & =\sum_{i=1}^{N} E_{i} D \cdot \cos \left(\sum_{n=1}^{i}\left(\phi_{\mathrm{nom}}-\phi_{n}\right)+\varphi_{\mathrm{inj}}\right) \\
& =\sum_{i=1}^{N} E_{i} D \cdot \cos \left(\sum_{n=1}^{i} \Delta \phi_{n}+\varphi_{\text {inj }}\right),
\end{aligned}
$$

where $\Delta \phi_{n}=\phi_{\text {nom }}-\phi_{n}$ is the deviation of the phase advance of the $n$ cell with respect to the nominal one. The sum $\phi_{\text {cum }}(i)=\sum_{n=1}^{i} \Delta \phi_{n}$ is the cumulative phase advance error along the structure.

Through Eq. (A3), it is possible to calculate the accelerating voltage for a given distribution of phase advances, and it is possible to compute the expected reduction in the average accelerating voltage due to the detuning of the structure simply defined as

$$
\begin{aligned}
\Delta V_{\mathrm{acc}} & =\frac{V_{\mathrm{acc}}-\sum_{i=1}^{N} E_{i} D \cdot \cos \varphi_{\mathrm{inj}}}{\sum_{i=1}^{N} E_{i} D \cdot \cos \varphi_{\mathrm{inj}}} \\
& =\frac{V_{\mathrm{acc}}}{\sum_{i=1}^{N} E_{i} D \cdot \cos \varphi_{\mathrm{inj}}}-1 .
\end{aligned}
$$

Assuming a perfect on-crest acceleration $\left(\varphi_{\text {inj }}=0\right)$, we report in Fig. 21(a), as an example, the distribution of the accelerating voltage variations calculated considering $10^{4}$ random cases. The random cases have a uniform distribution of the cell-to-cell phase advance with a maximum deviation of $\Delta \phi_{n}= \pm 2^{\circ}$ and a maximum cumulative phase advance error along the structure $\left(\phi_{\text {cum }}\right)$ within $\pm 5^{\circ}$. In the same plot, we also report the case of $\Delta \phi_{n}= \pm 5^{\circ}$ and $\phi_{\text {cum }} \pm 10^{\circ}$. From the plot, it evident that a tuning of the structure with the final cell-to-cell phase advance and the cumulative phase advance error in the first range allows 
one to keep the reduction of the accelerating voltage below $0.2 \%$.

[1] Technical design report EuroGammaS proposal for the ELI-NP gamma beam system, arXiv:1407.3669.

[2] K. Dupraz et al., Design and optimization of a highly efficient optical multipass system for $\gamma$-ray beam production from electron laser beam Compton scattering, Phys. Rev. Accel. Beams 17, 033501 (2014).

[3] T. Shintake, The Choke mode cavity, Jpn. J. Appl. Phys. 31, L1567 (1992).

[4] T. Shintake, HOM free linear accelerator structure using choke mode cavity, in Proceedings of the 17th Linear Accelerator Meeting, Sendai, Japan; Report No. KEKPREPRINT-92-66, National Laboratory for High Energy Physics 1-1 Oho, Tsukuba-shi, Ibaraki-ken, https://lss.fnal .gov/archive/other1/kek-preprint-92-66.pdf.

[5] T. Shintake, in Proceedings of the 15th Particle Accelerator Conference, PAC-1993, Washington, DC, 1993 (IEEE, New York, 1993).

[6] T. Inagaki, C. Kondo, H. Maesaka, T. Ohshima, Y. Otake, T. Sakurai, K. Shirasawa, and T. Shintake High-gradient $C$-band linac for a compact x-ray free-electron laser facility, Phys. Rev. Accel. Beams 17, 080702 (2014).

[7] L. Piersanti et al. Design, realization and test of C-band accelerating structures for the SPARC_LAB linac energy upgrade, Nucl. Instrum. Methods Phys. Res., Sect. A 837, 161 (2016).

[8] R. Zennaro et al., Measurement and high power test of the first C-Band Accelerating Structure for SwissFEL, in Proceedings of LINAC2014, Geneva, Switzerland (JACoW, 2014, p. 333.

[9] W. Fang et al., Design, fabrication and first beam tests of the C-band RF acceleration unit at SINAP, Nucl. Instrum. Methods Phys. Res., Sect. A 823, 91 (2016).

[10] D. Alesini et al., Design of high gradient, high repetition rate damped C-band rf structures, Phys. Rev. Accel. Beams 20, 032004 (2017).

[11] D. Alesini et al., Realization and high power tests of damped C-band accelerating structures for the ELI-NP linac, in Proceedings of IPAC2016, Busan, Korea (JACoW, 2016), MOPMW004, p. 399.

[12] A. Grudiev and W. Wuensch, Design of an X-band accelerating structure for the CLIC main linac, in Proceedings of LINAC08, Victoria, BC (2008), TRIUMF, p. 933.
[13] A. Grudiev and W. Wuensch, Design of the CLIC main linac accelerating structure for CLIC conceptual design report, in Proceedings of the 25th International Linear Accelerator Conference, LINAC-2010, Tsukuba, Japan (KEK, Tsukuba, Japan, 2010), p. 211.

[14] H. Zha and A. Grudiev, Design and optimization of Compact Linear Collider main linac accelerating structure, Phys. Rev. Accel. Bams 19, 111003 (2016).

[15] http://www.comeb.it/.

[16] D. Alesini, A. Citterio, G. Campogiani, L. Ficcadenti, M. Migliorati, A. Mostacci, L. Palumbo, S. Persichelli, and R. Zennaro, Tuning procedure for traveling wave structures and its application to the C-Band cavities for SPARC photo injector energy upgrade, J. Instrum. 8, P10010 (2013).

[17] http://www.morganbrazealloys.com/.

[18] G. De Michele et al., Broadband electromagnetic characterization of materials for accelerator components, in Proceedings of the 2nd International Particle Accelerator Conference, San Sebastián, Spain (EPS-AG, Spain, 2011), p. 769 .

[19] F. Cardelli, Ph.D. thesis, Sapienza University of Rome, Rome, 2017.

[20] https://www.ansys.com.

[21] http://research-instruments.de/.

[22] http://www.scandinovasystems.com/.

[23] X. Wu, J. Shi, H. Chen, J. Shao, T. Abe, T. Higo, S. Matsumoto, and W. Wuensch, High-gradient breakdown studies of an $X$-band Compact Linear Collider prototype structure, Phys. Rev. Accel. Beams 20, 052001 (2017).

[24] T. Argyropoulos et al., Design, fabrication, and highgradient testing of an $X$-band, traveling-wave accelerating structure milled from copper halves, Phys. Rev. Accel. Beams 21, 061001 (2018).

[25] A. Degiovanni, W. Wuensch, and J. G. Navarro, Comparison of the conditioning of high gradient accelerating structures, Phys. Rev. Accel. Beams 19, 032001 (2016).

[26] R. Zennaro, Design, construction and power conditioning of the first C-band test accelerating structure for SWISSFEL, in Proceedings of the 3rd International Particle Accelerator Conference, New Orleans, LA, 2012 (IEEE, Piscataway, NJ, 2012), p. 3329.

[27] T. P. Wangler, Principle of rf Linear Accelerators (Wiley., New York, 1998).

[28] E. Persico, E. Ferrari, and S. E. Segre, Principles of Particle Accelerators (W.A. Benjamin, New York, 1968). 